Collaborative learning between GPs, fledgling MDTs, and across the primary-secondary care interface has united colleagues around a shared purpose, starting solution-focused conversations.

\section{OUTCOMES OF THE ELECTRONIC MEDICAL TAKE LIST - IMPROVING STAFF EXPERIENCE, PATIENT SAFETY AND ROSTERING}

Alvin Shrestha, Alexia Pisinou, Craig Burke, Troy Haddon. Croydon University Hospital, UK; 3, 4 Patienteer

\subsection{6/leader-2020-FMLM.161}

Aims and Methods The 'medical take' is a demanding process, whereby doctors review and manage acute admissions. We created a task force group and collaborated with Patienteer, a patient tracking software that can pull real-time patient information from our electronic medical record system Cerner, in order to improve this experience for our staff and ultimately improve patient care.

A bespoke electronic medical take list was which allowed us to overcome previously identified issues:

- Patient details were automatically added to the list (removing the potential for transcription errors)

- Multiple user access

- Live location

- Live NEWS2 scores

Thereafter, 6 months' worth of data was analysed from February to September 2019.

Results Doctors were surveyed through an anonymised online questionnaire, with 27 respondents who had used both the old Excel list and the new Patienteer list. 89\% found Patienteer to be improve efficiency. Two-thirds overall found it helped prioritise clinical need. $78 \%$ found patient details were captured more accurately with Patienteer. Accessibility was also thought to have improved, with $85 \%$ favouring Patienteer as being 'easier' or 'much easier' to access. 59\% thought Patienteer had decreased their workload. 96\% would overall, recommend the new Patienteer medical take list.

Through 6344 patient encounters, various outcomes were reviewed. There was a significant inverse correlation between NEWS2 scores and time taken for a medical doctor review: as NEWS2 score increased, patients were seen earlier. The time taken to be seen in minutes was expressed as = 4.24621*NEWS +133.031 , where NEWS is the NEWS2 score $(\mathrm{p}=0.0002397)$.

Conclusion The survey suggested the Patienteer medical take list had made the acute medical take experience more efficient and decreased the workload. There is also a suggestion that patient care is improved through the display of the NEWS2 score, allowing doctors to prioritise sicker patients earlier.

\section{OPTIMISING THE CLINICAL PATHWAY FOR PLACENTAL HISTOLOGY}

${ }^{1}$ Nazia Din, ${ }^{1}$ Nicole Gentles, ${ }^{1}$ Benjamin Allison, ${ }^{1}$ Maria Hickland, ${ }^{2}$ Jilly Lloyd, ${ }^{2}$ Adam D Jakes. 'King's College London, UK; ${ }^{2}$ Guy's and St. Thomas' NHS Foundation Trust, London, UK

10.1136/leader-2020-FMLM.162
The Royal College of Pathologists (RCPATH) sets criteria for requesting placental histological examination which allows identification of pathological processes contributing to/causing an adverse obstetric outcome. Maternal intrapartum pyrexia is an essential criterion but is not part of the Guy's and St. Thomas' Trust (GSTT) guideline. There is no current baseline data on this at GSTT.

Aim To review current practice of requesting placental histology and amend trust guidelines to follow national recommendations.

Birth records from June 2019 were reviewed showing $8 \%(47 / 569)$ of deliveries were eligible for placental histology as per trust criteria but only 60\%(28/47) were requested. A survey demonstrated $4 \%$ of staff were able to correctly identify all criteria for histology and there was confusion regarding formalin use. Neonatologists and pathologists were contacted to identify views on the usefulness of placental histology and the effect of adopting national criteria.

The pathologists confirmed all placentas should be sent in formalin unless specified by the obstetric team. The intrapartum infection guideline was amended to include requesting placental histology after approval by the intrapartum committee. The obstetric theatre posters were amended to include the extended criteria. A training presentation was emailed to all staff on the procedure for requesting placental histology.

The trust guidelines were successfully amended to include RCPATH criteria for placenta histology. Birth records from January 2020 identified 13\%(63/487) of deliveries were eligible for placental histology, but only 56\%(35/63) were requested.

The trust guideline for requesting placental histology now follows national recommendations. This may provide answers for adverse obstetric outcomes and reduce trust medicolegal costs. Unfortunately, no improvement in placental histology requests was demonstrated. Exploring potential barriers to improvement will be part of the next change cycle.

\section{QUALITY IMPROVEMENT PROJECT ON COMMUNITY INDUCTION FOR FOUNDATION YEAR 2 DOCTORS}

${ }^{1} \mathrm{H}$ Minali Perera, ${ }^{2}$ Victoria Apel, ${ }^{2}$ Katie Chu. ${ }^{1}$ Princess Alexandra Hospital, Harlow; ${ }^{2}$ Epsom and St Helier Hospital NHS Trust, Surrey

\subsection{6/leader-2020-FMLM. 163}

Foundation doctors regularly rotate through different specialties as part of their training. Inductions to these new placements can vary in delivery and effectiveness. High quality induction is a recommendation of the Academy of Medical Royal Colleges for safe trainee changeover.

Aim To improve the induction process for Foundation Year 2 doctors (FY2s) in community placements at Epsom and St Helier Trust.

A survey of 10 questions, devised from the British Medical Association guidance on adequate induction, was circulated to FY2 doctors $(n=10)$ in community placements (GP and Psychiatry) between 4th August - 4th December 2019. Induction booklets individualised to each placement were created by 